\title{
Bilateral postoperative maxillary cysts presenting as a diagnostic challenge
}

\author{
Sel Ae Hwang, Byung-Cheol Kang, Suk-Ja Yoon, Liu Liu, Jae-Seo Lee* \\ Department of Oral and Maxillofacial Radiology, School of Dentistry, Chonnam National University, Gwangju, Korea
}

\begin{abstract}
Postoperative maxillary cysts (POMCs) are considered as a delayed complication, which occurs after several years of occurring many years after maxillary sinus surgery. The variable symptoms related to POMCs include swelling and pain in the buccal region, discomfort in the maxilla or maxillary teeth, and exophthalmos. Symptoms related to the teeth may be confused with those of an odontogenic disease. Herein, We report about the delayed diagnosis of bilateral POMCs in the maxillary region 30 years after CaldwellLuc surgery for maxillary sinusitis.
\end{abstract}

Key Words: Postoperative complications, Maxillary sinus, Cyst

(c) This is an open-access article distributed under the terms of the Creative Commons Attribution Non-Commercial License (http://creativecommons.org/licenses/by-nc/4.0) which permits unrestricted noncommercial use, distribution, and reproduction in any medium, provided the original work is properly cited.

\section{INTRODUCTION}

Postoperative maxillary cysts (POMCs), which are also known as surgical ciliated cysts, were first described by Kubo in 1927 [1]. POMCs develop as a delayed complication many years after a surgical procedure such as radical sinus surgery [1,2], orthognathic surgery [3,4], sinus elevation [5], and midfacial fracture surgery [6]. Entrapment of the sinus epithelial lining within the bone can occur during the healing process after a surgical procedure and the lesion grows as an epithelial-lined cavity $[7,8]$.

The variable symptoms related to POMCs include swelling and pain in the buccal and infraorbital regions $[2,3,5]$, discomfort in the maxilla or maxillary teeth $[2,4]$, and exophthalmos [9].

The radiographic features of POMCs include a unilocular or multilocular radiolucency with a well-defined border. Large POMCs cause bony expansion, displacement, or perforation of adjacent sinus walls and invasion of the surrounding tissue [2,4].

When a lesion appears in the region of the posterior maxillary teeth, the patient may complain of pain in the teeth; the resulting lesion may thus be confused as an odontogenic disease [8]. Furthermore, when lesions are located in both maxillary sinuses, the appearance may resemble that of normal maxillary sinuses. Previous studies have shown the difficulty in detecting POMCs when the lesion is limited to the maxillary sinus [10,11]. Lack of awareness regarding POMCs may lead to misdiagnosis and delayed management. We have reported on a case of delayed diagnosis of bilateral POMCs before definitive treatment.

Received June 12, 2018; Revised July 17, 2018; Accepted August 1, 2018

Corresponding author: Jae-Seo Lee, Department of Oral and Maxillofacial Radiology, School of Dentistry, Dental Science Research Institute, Chonnam National University, 77 Yongbong-ro, Buk-gu, Gwangju 61186, Korea.

Tel: +82-62-530-5686, Fax: +82-62-530-5679, E-mail: jsyi16@hanmail.net

Copyright $\odot$ 2018, Oral Biology Research Institute 


\section{CASE}

A 56-year-old woman presented to the Department of Conservative Dentistry at Chonnam National University Dental Hospital, Gwangju, Korea. She complained of tenderness on palpation of the left maxillary vestibule. On intra-oral examination, only generalized mild calculus deposition was identified. No swelling or fluctuation was observed.

Periapical radiographic examination (CS6100; Carestream, Rochester NY, USA) and panoramic radiographic examination (Kodak 8000C; Carestream) were performed. Radiographic examinations showed apical radiolucency at the apex of \#25 and generalized mild alveolar bone loss (Fig. 1). The clinical diagnosis was a periapical abscess at \#25, and root canal therapy was performed. Her first complaint was relieved, but she complained of percussion pain at \#17, and further root canal therapy was performed. However, rather than being relieved, her pain was exacerbated. She complained of spontaneous pain with sensitivity to cold and hot in the right maxillary posterior teeth. Furthermore, swelling of the right cheek occurred. Because of percussion pain at \#16, further root canal therapy was performed. However, her symptoms were not relieved and referred pain to the temporomandibular joint occurred.

Because of her persistent pain, an endodontist considered the possibility that the lesion was not due to an odontogenic disease but due to maxillary sinus disease. The patient's medical history was meticulously retaken, revealing that she had undergone Caldwell-Luc surgery
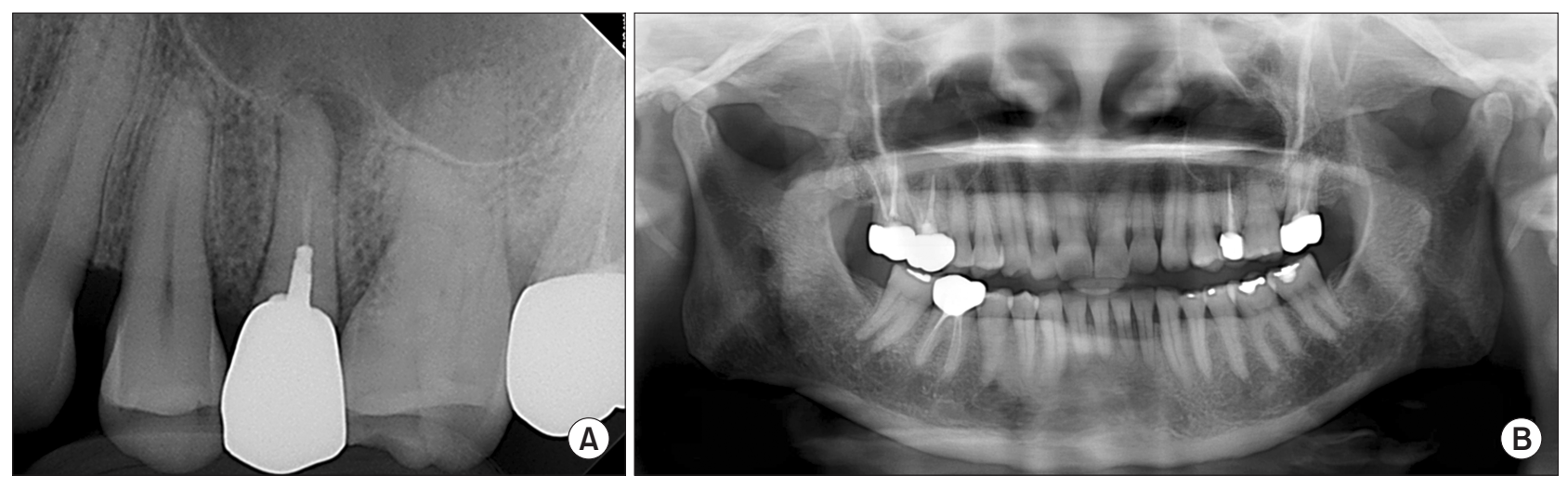

Fig. 1. (A) Periapical image showing a radiolucent lesion at the apical region of \#25. (B) Panoramic image showing well-defined round radiolucencies with cortical borders in each side of the maxillary sinus.
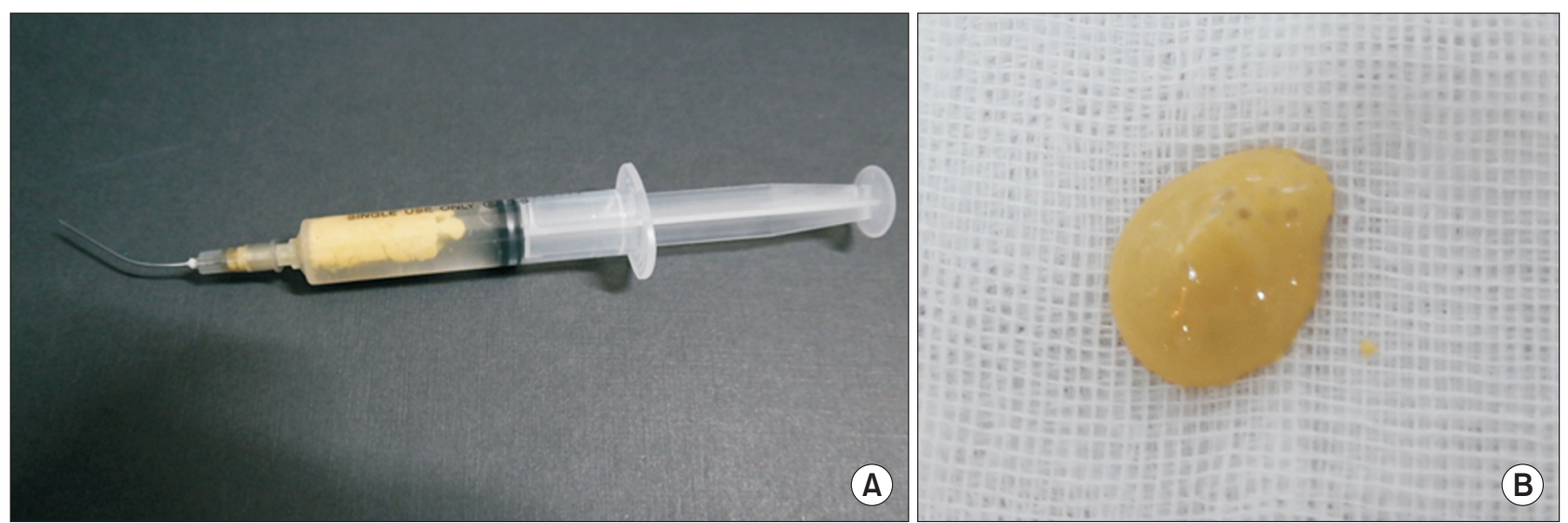

Fig. 2. (A) Aspiration was performed through the right maxillary sinus anterior wall; (B) $2 \mathrm{~mL}$ of yellow and thick fluid pus with foul odor was acquired. 
for treatment of bilateral maxillary sinusitis 30 years previously. Panoramic imaging was reinterpreted and welldefined round radiolucencies with cortical borders were detected bilaterally, limited to the sinuses. The lesions on the panoramic images must have been overlooked because of their symmetric morphology, leading to a misdiagnosis of normal maxillary sinuses.

Fluctuation was detected on palpation of the right vestibule, and aspiration was performed through the right sinus anterior wall; $2 \mathrm{~mL}$ of yellow and thick fluid pus with foul odor was acquired (Fig. 2). To establish the exact boundary and shape of the cyst, $1.5 \mathrm{~mL}$ of contrast media (Iopamiro 370; Bracco, Milan, Italy) was injected before radiographic images were obtained. On panoramic images, welldefined unilocular round lesions were noted. On computed tomography (CT) images (DiscoveryCT750; GE, Milwaukee, Wisconsin, USA), both sides of the lesions were limited to the sinuses. The right maxillary sinus anterior wall was thinned, which enabled aspiration. In contrast, the left maxillary sinus showed neither thinning nor expansion of
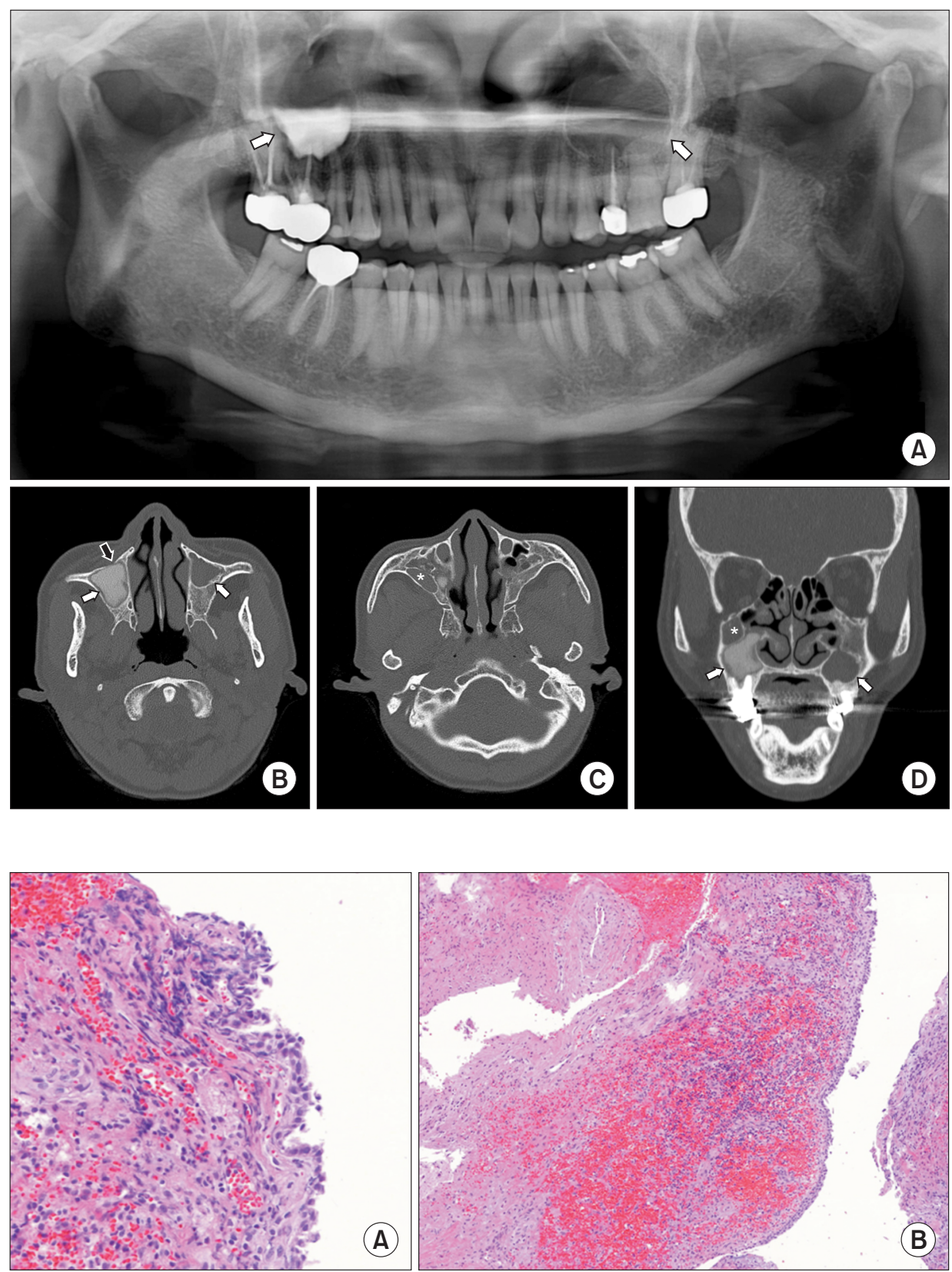

(A)

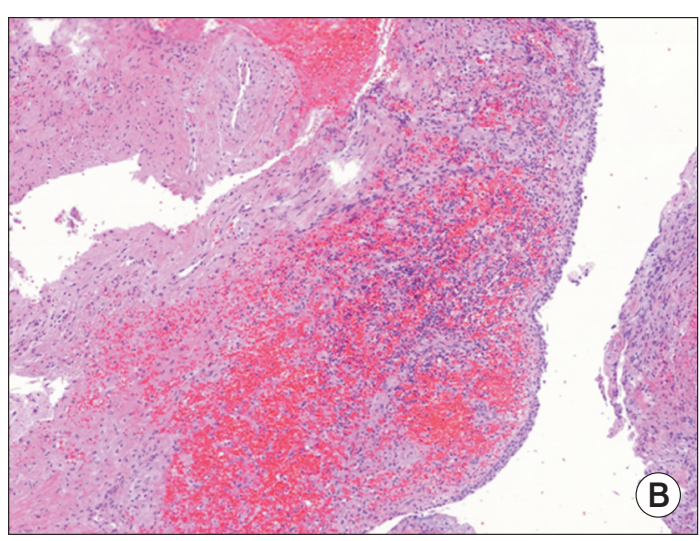

Fig. 3. Panoramic and computed tomography $(\mathrm{CT})$ images after administration of contrast medium. (A) Well defined bilateral cystic lesions (white arrow) in each side of the maxillary sinus. (B, C) Axial CT images showed bilateral cystic lesions (white arrow) in each side of the maxillary sinus. Anterior wall perforation of the right maxillary sinus (black arrow) was noted. A secondary cyst $\left(^{*}\right)$ was detected and isolated from the right postoperative maxillary cyst (POMC). (D) The coronal image showed the location of the secondary cyst $\left(^{*}\right)$ in the lateral portion of the right orbital floor. The right side POMC extended to the nasal cavity and involved the tooth.

Fig. 4. (A) Histopathological examination showing true cystic structures lined by a pseudostratified ciliated columnar epithelium (H\&E staining). (B) Infiltrating inflammatory cells and squamous metaplasia are seen around the cystic cavity (H\&E staining). 
any bone. No internal calcifications or sclerotic changes of the wall lining were observed on either side. A secondary cyst was detected on CT images. The secondary cyst was located in the right orbital floor lateral portion, isolated from the POMC (Fig. 3).

The patient visited the ears, nose, and throat department of Chonnam National University Hwasun Hospital (Hwasun, Korea) to undergo surgery. The surgery was performed using the inferior meatal antrostomy (IMA) method with local anesthesia. The cystic fluid containing necrotic tissue and brownish fluid was drained. The secondary cyst in the right orbital floor was also opened. The day after surgery, facial swelling and pain were relieved and the toothache she had felt for 6 months had also subsided.

Photomicrographs showed infiltrated inflammatory cells. The lesions exhibited true cystic structures lined by a pseudostratified ciliated columnar epithelium and squamous metaplasia (Fig. 4). These microscopic examination findings were consistent with the POMCs. Finally, the lesions were diagnosed as bilateral POMCs.

\section{DISCUSSION}

In this case, 6 months have passed since the patient's first visit to the final diagnosis of bilateral POMCs. Some factors made diagnosis difficult. First, meticulous history taking was not performed at the first visit. Second, the patient experienced symptoms mimicking pain of odontogenic origin. Moreover, periapical lesions actually existed at the pain site. Third, the lesions on panoramic images were overlooked because of their symmetric bilateral morphology and their location being limited to the sinus, without any perforation or expansion of the bone. Because of these factors, the lesions were not detected.

Heo et al. [11] found that observers failed to detect lesions limited to the sinus, for example, those on the floor of the maxillary sinus without any apparent bony expansion or those only on the anterior wall when bony expansion was observed. A previous study [10] showed that it was difficult for all observers to detect POMCs limited to the sinus, even for oral radiology specialists.

Panoramic radiography is the imaging modality generally used to detect maxillary sinus abnormalities [10] and offers a broad view of the sinus, as well as parts of the inferior, posterior, and anteromedial walls $[12,13]$. However, Ohba et al. [14] reported that panoramic radiography is less effective for detecting lesions located on the anterior and middle sinus walls. Gang et al. [10] reported that POMC lesions limited to the sinus are difficult to detect in panoramic views. Overlapping of adjacent anatomic structures and ghost images occurring in panoramic images limits their utility [12].

Advanced imaging modalities, such as CT and Cone beam $\mathrm{CT}$, are additionally recommended for further evaluation of POMCs. These imaging modalities can provide multiplanar images of the sinus and may contribute considerably to delineating the extent of disease and evaluating the loculation. Coronal images provide superior visualization of the ostiomeatal complex and nasal cavities and can identify any reaction in the surrounding bone to sinus diseases [12].

Moreover, some reports have suggested that magnetic resonance (MR) imaging should be considered as an additional imaging modality in POMC cases because the borders and extensions of cysts and cystic content as well as the presence of secondary cysts are more easily observed on MR images compared with CT images [15].

Contrast radiography was performed to determine whether the radiolucent cavity was suggestive of cyst or normal maxillary sinus, and to define the boundary of the lesion. A previous study showed that this technique contributed to locating the lesion and defining its boundary [16]. Positive aspiration and injection of contrast media in the right maxillary sinus revealed that well-defined, round radiolucency with cortical border was not maxillary sinus but cystic lesion.

In this study, after injecting contrast media the posterior boundary of the lesion was observed more clearly in panoramic image. In addition, as the boundary of the lesion became clear, secondary cyst could be easily found on CT images.

Full evaluation of a lesion makes it possible to plan appropriate surgical methods, including external approaches, marsupialization, Caldwell-Luc procedures, and endoscopic surgery [15]. Endoscopic intranasal surgery has recently increased in popularity [17]. The use of the endoscopic approach makes regeneration of normal 
mucosa easier, eliminates the need for an external incision, leads to less bleeding, and takes less time for surgery and recovery [18]. In particular, IMA provides superior mucociliary drainage and makes mucosal change reversion noticeable even after inferior meatal window closure has been performed [19]. However, some authors have expressed reservations about the endoscopic treatment of POMC, particularly when the lesion extends outside the sinus because drainage can fail, possibly leading to recurrence [20]. The patient in our study had lesions imitating the normal sinus, located near to the nasal cavity and suitable for endoscopic surgery, which achieved aeration and drainage of the POMC cavity through a wide IMA.

Lack of awareness of POMC may lead to misdiagnosis and delayed management. In this study, it took 6 months to diagnose a $\mathrm{POMC}$, resulting persistent pain before definite treatment was administered. Therefore, to diagnose POMC when patients complain of posterior maxillary tooth pain, history taking for surgery at the first evaluation is essential. POMCs can develop up to 49 years after initial surgery of the maxillary sinus [21]. Clinicians should ask patients specifically about their lifetime history of maxillofacial surgery, such as radical sinus surgery, orthognathic surgery, sinus elevation, and trauma.

Although bilateral POMC is rarely encountered, clinicians should be aware of such lesions and must be able to diagnose them at the earliest stage.

\section{CONFLICTS OF INTEREST}

The authors declare that they have no competing interests.

\section{ORCID}

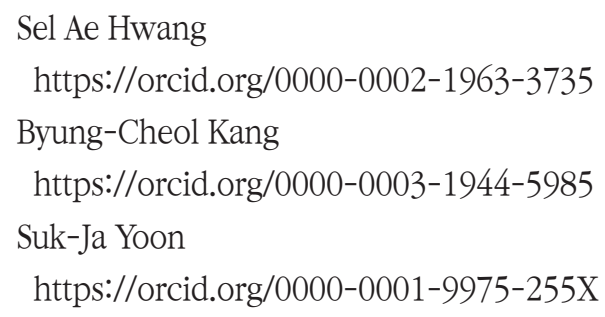

Liu Liu

https://orcid.org/0000-0001-5766-5351

Jae-Seo Lee

https://orcid.org/0000-0001-8059-0378

\section{REFERENCES}

1. Kubo I. A buccal cyst occurred after a radical operation of the maxillary sinus. J Otol Tokyo 1927;33:896-897.

2. Pe MB, Sano K, Kitamura A, Inokuchi T. Computed tomography in the evaluation of postoperative maxillary cysts. J Oral Maxillofac Surg 1990;48:679-684. doi: 10.1016/0278-2391(90)90049-8.

3. Sugar AW, Walker DM, Bounds GA. Surgical ciliated (postoperative maxillary) cysts following mid-face osteotomies. Brit J Oral Maxillofac Surg 1990;28:264-267. doi: 10.1016/0266-4356(90)90064-R.

4. Amin M, Witherow H, Lee R, Blenkinsopp P. Surgical ciliated cyst after maxillary orthognathic surgery: report of a case. J Oral Maxillofac Surg 2003;61:138-141. doi: 10.1053/joms.2003.50050.

5. Lockhart R, Ceccaldi J, Bertrand JC. Postoperative maxillary cyst following sinus bone graft: report of a case. Int J Oral Maxillofac Implants 2000;15:583-586.

6. An J, Zhang Y. Surgical ciliated cyst of the medial canthal region after the management of a midfacial fracture: a case report. J Craniofac Surg 2014;25:701-702. doi: 10.1097/ SCS.0000000000000675.

7. N Amin, T Pustylnik, J Jakubowski. Surgical ciliated cyst of the maxilla secondary to a prior sinus exposurecase report and literature review. Oral Surg Oral Med Oral Pathol Oral Radiol 2014;118:e188. doi: 10.1016/j. oooo.2014.05.077.

8. Leung YY, Wong WY, Cheung LK. Surgical ciliated cysts may mimic radicular cysts or residual cysts of maxilla: report of 3 cases. J Oral Maxillofac Surg 2012;70:e264-e269. doi: 10.1016/j.joms.2011.12.022.

9. Ohba T, Yang RC, Chen CY, Uneoka M. Postoperative maxillary cyst. Int J Oral Surg 1980;9:480-483. doi: 10. 1016/S0300-9785(80)80080-9.

10. Gang TI, Huh KH, Yi WJ, Lee SS, Heo MS, Choi SC. The effect of radiographic imaging modalities and the observer's experience on postoperative maxillary cyst assessment. Imaging Sci Dent 2014;44:301-305. doi: 10.5624/isd. 2014.44.4.301.

11. Heo MS, Song MY, Lee SS, Choi SC, Park TW. A comparative study of the radiological diagnosis of postoperative maxillary cyst. Dentomaxillofac Radiol 2000;29:347-351. doi: $10.1038 / \mathrm{s} / \mathrm{dmfr} / 4600552$.

12. White SC, Pharoah MJ. Oral radiology: principles and interpretation. St.Louis: Mosby/Elsevier; 2014. 
13. Ohba T, Katayama H. Panoramic roentgen anatomy of the maxillary sinus. Oral Surg Oral Med Oral Pathol 1975;39: 658-664. doi: 10.1016/0030-4220(75)90211-X.

14. Ohba T, Ogawa Y, Hiromatsu T, Shinohara Y. Experimental comparison of radiographic techniques in the detection of maxillary sinus disease. Dentomaxillofac Radiol 1990;19: 13-17. doi: 10.1259/dmfr.19.1.2387471.

15. Chindasombatjaroen J, Uchiyama Y, Kakimoto N, Murakami S, Furukawa S, Kishino M. Postoperative maxillary cysts: magnetic resonance imaging compared with computerized tomography. Oral Surg Oral Med Oral Pathol Oral Radiol Endod 2009;107:e38-e44. doi: 10.1016/ j.tripleo.2009.02.012.

16. Makandar AM, Kshar A, Byakodi R. Use of contrast radiography to differentiate unilocular cystic lesion from multilocular one-a case report. Oral Surg Oral Med Oral Radiol 2014;2:1-5. doi: 10.12691/oral-2-1-1.

17. Busaba NY, Salman SD. Maxillary sinus mucoceles: clinical presentation and long-term results of endoscopic surgical treatment. Laryngoscope 1999;109:1446-1449. doi: 10. 1097/00005537-199909000-00017.

18. Ikeda K, Hirano K, Oshima T, Shimomura A, Suzuki $\mathrm{H}$, Sunose H, Kondo Y, Takasaka T. Comparison of complications between endoscopic sinus surgery and Caldwell-Luc operation. Tohoku J Exp Med 1996;180:2731. doi: 10.1620/tjem.180.27.

19. Mann W, Beck C. Inferior meatal antrostomy in chronic maxillary sinusitis. Arch Otorhinolaryngol 1978;221:289295. doi: 10.1007/BF00491465.

20. Marks SC, Latoni JD, Mathog RH. Mucoceles of the maxillary sinus. Otolaryngol Head Neck Surg 1997;117:1821. doi: 10.1016/S0194-5998(97)70200-6.

21. Bulut AŞ, Şehlaver C, Perçin AK. Postoperative maxillary cyst: a case report. Patholog Res Int 2010 Nov 4 [Epub]. http://doi.org/10.4061/2010/810835. 